\title{
DGC-specific RHOA mutations maintained cancer cell survival and promoted cell migration via ROCK inactivation
}

\author{
Takashi Nishizawa ${ }^{1}$ Kiyotaka Nakano ${ }^{1}$, Aya Harada ${ }^{1}$, Miwako Kakiuchi², Shin-Ichi \\ Funahashi $^{1}$, Masami Suzuki ${ }^{1}$, Shumpei Ishikawa ${ }^{2,3}$ and Hiroyuki Aburatani ${ }^{2}$ \\ ${ }^{1}$ Department for Research, Forerunner Pharma Research Co., Ltd., Tokyo, Japan \\ ${ }^{2}$ Genome Science Division, Research Center for Advanced Science and Technology, The University of Tokyo, Tokyo, Japan \\ ${ }^{3}$ Department of Genomic Pathology, Medical Research Institute, Tokyo Medical and Dental University, Tokyo, Japan \\ Correspondence to: Takashi Nishizawa, email: nishizawatks@chugai-pharm.co.jp
}

Keywords: RHOA; ROCK; diffuse gastric cancer; mutation; ARHGAP

Received: November 26, 2017 Accepted: April 06, 2018

Published: May 01, 2018

Copyright: Nishizawa et al. This is an open-access article distributed under the terms of the Creative Commons Attribution License 3.0 (CC BY 3.0), which permits unrestricted use, distribution, and reproduction in any medium, provided the original author and source are credited.

\section{ABSTRACT}

RHOA missense mutations exist specifically in diffuse type gastric cancers (DGC) and are considered one of the DGC driver genes, but it is not fully understood how RHOA mutations contribute to DGC development. Here we examined how RHOA mutations affect cancer cell survival and cell motility. We revealed that cell survival was maintained by specific mutation sites, namely G17, Y42, and L57. Because these functional mutations suppressed MLC2 phosphorylation and actin stress fiber formation, we realized they act in a dominant-negative fashion against the ROCK pathway. Through the same inactivating mechanism that maintained cell survival, RHOA mutations also increased cell migration activity. Cell survival and migration studies on CLDN18-ARHGAP (CLG) fusions, which are known to be mutually exclusive to RHOA mutations, showed that CLG fusions complemented cell survival under RHOA knockdown condition and also induced cell migration. Site-directed mutagenesis analysis revealed the importance of the GAP domain and indicated that CLG fusions maintained RHOA in the inactive form. Taken together, these findings show that the inactivation of ROCK would be a key step in DGC development, so ROCK activation might provide novel therapeutic opportunities.

\section{INTRODUCTION}

Diffuse-type gastric cancers (DGC) account for approximately $30 \%$ of all gastric cancers and are characterized by poorly differentiated adenocarcinoma with a worse prognosis than the intestinal type [1-3]. DGC infiltrate into adjacent stromal tissues, spread without clear polyps or ulcers, and frequently show intraperitoneal metastasis [4, 5]. Comprehensive genomic sequencing studies to identify DGC-specific genetic alterations, including our previous study, have shown that $14-25 \%$ DGC patients carry $R H O A$ missense mutations, such as R5W, G17E, Y42C, and L57V [6-8].

RHOA is a small GTPase that belongs to the RHO family and has various biological functions, such as cytokinesis, cell motility, and tissue development [9-11]. RHOA cycles between the GDP-bound inactive form and the GTP-bound active form under the control of regulatory proteins like guanine nucleotide exchange factors (GEFs) and GTPase-activating proteins (GAPs). These regulatory proteins induce conformational change in RHOA to allow binding to substrates named effector proteins, one of which is Rho-associated protein kinase (ROCK). ROCK-LIMK-CFL1 signaling contributes to actin filament stabilization, while ROCK-MLCP-MLC signaling promotes actomyosin formation $[12,13]$.

In our previous work, we observed that a knockdown of RHOA in RHOA-mutated cancer cell lines represses cell survival significantly [6]. Wang et al. also reported that introducing RHOA mutations, Y42 and $\mathrm{L} 57 \mathrm{~V}$, to a murine intestinal organoid promotes cell survival [7]. Moreover, a comprehensive investigation of TCGA revealed that negative regulators of RHOA, GAP6 and GAP26, fused with the tight junction membrane protein $C L D N 18$ in a DGC-specific manner 
[8]. The frequency of CLDN18-GAP (CLG) fusions is $15 \%$ in DGC and, interestingly, RHOA mutations and $C L G$ fusions are mutually exclusive. Although these results suggest that a dysregulated RHOA signal is related to DGC development, the details remain to be understood. In this study, we explored the contribution of RHOA mutations to DGC development, focusing on cell survival and also on cell motility, which is one of the features of DGC. Furthermore, we evaluated the functional relationship between RHOA mutations and $C L G$ fusions.

\section{RESULTS}

\section{RHOA-siRNA treatment inhibited $3 \mathrm{D}$ cell survival of $R H O A$-mutated cell lines in a mutation-dependent manner}

To identify which mutation sites contribute to cancer cell survival, we selected RHOA-mutated cell lines from public databases (Supplementary Table 1) and chose 12 cell lines. In 3D culture conditions, we evaluated the inhibition efficacy of RHOA-siRNAs on cell survival and observed significant RHOA-siRNAdependent inhibition in cell lines HCC95, SW948, BT474, and OE19, which carried G17 or Y42 single mutations (Figure 1). On the other hand, cell survival inhibition seen in R5, Y34, E40, A61, and A69 single mutants or in R5/Y42 or R5/F39 double mutants was less clear, and cell survival of 4 RHOA-WT cell lines was not inhibited (Supplementary Figure 1).

\section{Mutated $R H O A$ contributed cell survival, and G17V, Y42C, Y42S, and L57V mutations showed functional complementarity to G17E}

Next, we investigated which types of $R H O A$ contribute to cell survival in SW948 cells, which express G17E- and WT-RHOA heterogeneously. We used stable SW948 transfectants that expressed siRNA-resistant G17E- and WT-RHOA, and then evaluated whether RHOA-siRNA continued to inhibit cell survival or not (Figure 2). While the introduced G17E mutation restored cell survival, the WT did not.

We also checked the functional complementarity with mutations that were found in clinical specimens. Because L57V-mutated cancer cell lines were unavailable commercially, we added the mutation for this experiment. The siRNA-dependent inhibition of cell survival was cancelled not only by the introduction of G17E, but also of G17V, Y42C, Y42S, and L57V; however, it was not cancelled by the R5W mutant (Figure 2). To confirm these results, we also expressed abundant mutated $R H O A$ transiently in SW948 to evaluate cell survival, and the same tendency was observed (Supplementary Figure 2B).
These results revealed that the mutations in G17, Y42, and L57 also contributed to cancer cell survival.

\section{RHOA-knockdown in $R H O A$-mutated SW948 induced ROCK activation via RHOB}

To reveal the signal cascade that contributes to cell survival, we analyzed the time course of the ROCK pathway, which is one of the major RHOA signaling pathways, after RHOA-siRNA treatment. We evaluated the change in other RHO family proteins, RHOB and RHOC, and in signal molecules, ROCK1/2, MLC2, MYPT1, LIMK1/2, and CFL1. RHOA protein expression was knocked down significantly on Day 1 after RHOAsiRNA treatment and was almost completely depleted on Day 2 (Figure 3A), while the expression of RHOB and RHOC proteins was accordingly elevated. $R H O A-$ siRNA treatment elevated the phosphorylation of MLC2 (Thr18/Ser19). We also noted that MYPT1 (Thr696 and Thr853), which is a phosphatase of MLC2, was not phosphorylated (Supplementary Figure 3), and LIMK1 (Thr508)/LIMK2 (Thr505) and CFL1 (Ser3) were constantly phosphorylated independently of RHOAsiRNA. From these results, we assumed that RHOA depletion induced ROCK-MLC2 signal activation. To clarify whether the ROCK activation induced by RHOA depletion affected the cytoskeleton or not, we stained for actin stress fiber. After RHOA-siRNA treatment, the formation of actin stress fiber was clearly increased and the shape was spiky (Figure 3B). This result verified that a knockdown of RHOA activated ROCK and stimulated actin stress fiber formation. Next, to investigate whether the suppression of ROCK would promote cell survival or not, we evaluated the effect of a ROCK $1 / 2$ inhibitor, Y-27632, on the cell survival of SW948. After treatment with Y-27632, the survival rate of RHOA-siRNAtreated cells recovered significantly from $24 \%$ (nontreatment) to $82 \%(3 \mu \mathrm{M})$ and $92 \%(10 \mu \mathrm{M})$ (Figure $3 \mathrm{C})$. Y-27632 also inhibited the phosphorylation of MLC2 (Supplementary Figure 4). We revealed that inactivation of ROCK promoted cell survival. Overall, this series of results revealed that $R H O A$ mutations keep suppressing ROCK activation, so their effect on ROCK is dominantnegative.

We hypothesized that ROCK reactivation would be induced by RHOB and/or RHOC, because the expression of these RHO molecules was elevated after RHOA-siRNA treatment. To evaluate this hypothesis, we used $R H O B$ and/or $R H O C$-siRNAs for a rescue study. The survival rate of RHOA-siRNA-treated cells increased significantly from $13 \%$ to $61 \%$ ( + RHOB- and RHOC-siRNAs), $59 \%$ $(+R H O B$-siRNA) and $24 \%(+R H O C$-siRNA) (Figure 3D). $R H O B$-siRNA inhibited the phosphorylation of MLC2 induced by RHOA-siRNA treatment (Figure 3E). These results revealed that ROCK activation was induced by RHOB in SW948. 


\section{The inhibition of cell survival by $R H O A$-siRNA was cancelled by $C L G$ fusions and $G A P s$, but not by $C L D N 18$}

To reveal the functional relationship between $R H O A$ mutations and $C L G$ fusions, we treated stable SW948 transfectants that expressed $C L G$ fusions with $R H O A$ siRNA and evaluated the effect on cell survival. The domain structures of CLDN18, GAP6, GAP26, CLG6, and CLG26 are shown in Figure 4A. RHOA-siRNA-dependent cell survival inhibition was canceled by CLG6, CLG26, GAP6, and GAP26, but not by CLDN18 (Figure 4B). These results indicated that $C L G$ fusions complemented RHOA mutations, in terms of their effect on cell survival.

Further investigation served to confirm whether survival in cells with $C L G$ fusions was dependent on GAP, which inactivates $R H O A$. A published report indicated the intensity of the RHOA and GAP26 interaction by showing that mutations of K454 and R458 in GAP26 remarkably decreased the thermodynamic and kinetic scores [14]. Using 3D modeling, we confirmed that K454 and R458 are important for the interaction between GAP26 and RHOA (Figure 4C) because they form hydrogen bonds to D65 in RHOA. Therefore, we introduced K454A/R458E double-mutated CLG26 into SW948 and established a stable transfectant in which GAP activity was eliminated. This double mutation in the GAP domain significantly decreased the contribution of CLG26 to survival (Figure 4D). These results suggested that GAP activity was necessary for cell survival.

\section{RHOA mutations and $C L G$ fusions induced migration activity by inactivating ROCK}

Next, we evaluated the effect of RHOA mutations on cell motility, which is a feature of DGC. We introduced WT, G17E, Y42C, and Y42S into MKN74, and used the transfectants for migration and invasion assays in a Boyden Chamber. Compared with mock, G17E, Y42C, and $\mathrm{Y} 42 \mathrm{~S}$ promoted cell migration activity $1.6-$ to $2.0-$ fold, whereas WT decreased the migration activity 0.65 fold (Figure 5A). Representative images of the migration assay are shown in Supplementary Figure 5. We also evaluated the invasion activity of these transfectants with a Matrigel-coated chamber, but a clear difference was not observed (Figure 5B).

When we evaluated cell motility in $C L G$ fusions, migration activity compared with mock was significantly increased by CLG6, CLG26, GAP6, and GAP26, but not by CLDN18 (Figure 5C). However, when the K454A/ R458E double mutation in the GAP domain was introduced to the CLG26 transfectant, the migration activity was diminished (Figure 5D). We revealed that, similarly to RHOA mutations, $C L G$ fusions contributed to migration activity in addition to cell survival, and that this contribution was dependent on the GAP activity.
As in the cell survival assays, we clarified the relationship between cell migration and ROCK activation by staining for actin stress fiber to reveal the cytoskeleton of MKN74 transfectants (Figure 5E). The mock transfectant showed clear stress fiber formation localized around cell clusters; on the other hand, G17E, Y42C, and Y42S showed weaker actin stress fiber formation, and their localization around cell clusters was unclear. These cytoskeletal changes induced by RHOA mutations were similar to those found in Y-27632-treated cells, which indicates the possibility that the inactivation of ROCK also contributed to migration activity. To verify this hypothesis, we evaluated the migration activity of MKN74 after Y-27632 treatment. As a result, Y-27632 increased migration activity 2.1 -fold $(3 \mu \mathrm{M})$ and 2.9 -fold $(10 \mu \mathrm{M})$ compared with non-treatment (Figure 5F). These results revealed that the inactivation of ROCK promoted cell migration activity.

\section{DISCUSSION}

In this study, we revealed that $R H O A$ mutations promoted cancer cell survival and migration activity by inactivating ROCK. At first, the presence of several hotspot amino acids led us to assume that RHOA mutations would be gain-of-function mutations, similar to mutations in $R A S$ and $R A C$ [15-18]. However, contrary to our expectations, the knockdown of $R H O A$ induced ROCK activation, and a ROCK inhibitor achieved cell survival similar to that seen in RHOA mutations; therefore, we concluded that functional RHOA mutations were lossof-function (LoF) mutations for ROCK activation. Wang et al. reported that the amount of GTP form of RHOA in $\mathrm{Y} 42 \mathrm{C}$ and L57V was less than that in WT and G14V in a pull-down assay [7], which would support our conclusion. Although $R B 1$ and $V H L$ are well known as tumor suppressor genes that have LoF mutations, they have no clear hotspots [19]. Despite the presence of hotspots, the RHOA mutations were LoF type, and cell lines acquired dominant-negative features when site-specific mutations were introduced. Our analysis demonstrated that the hotspot mutations at G17, Y42, and L57 contributed to cell survival, but not those at R5 and L69. On the other hand, in Burkitt's lymphoma, R5 mutation was reported to be a hotspot and to suppress RHOA-ROCK signaling [20], which suggests that the mechanism by which RHOA mutations induce dominant-negative properties might vary depending on the tumor type or cell type. Our next challenge will be to clarify the mechanism by which each RHOA mutant inactivates ROCK signaling in DGC.

Our study provided interesting insights about the mechanism of cell death by RHOA knockdown. Firstly, RHOA knockdown reactivated the ROCK pathway mainly via RHOB. We observed that the protein expression of RHOB and/or RHOC was induced in other cell lines besides SW948 (Supplementary Figure 6), as have other 
groups [21], which suggests that homeostasis to keep the total amount of RHOs is generally maintained. RHOs have been previously reported to complement each other functionally [22, 23]. Mutated RHOA suppresses ROCK activation, but it was interesting to see that upregulated RHOB after RHOA knockdown revitalized ROCK signaling through this complementary mechanism. Secondly, ROCK activation induced cell death in $R H O A$ mutated cancer cells. In human ES cells and iPS cells, it has been reported that inactivating the ROCK pathway significantly enhances recovery of cells from cryopreserved stocks in cell culture [24]. Upon dissociation, these cells become vulnerable to apoptosis via a phenomenon called apoptotic membrane blebbing. The molecular mechanism that causes apoptotic membrane blebbing would be ROCK signaling activation [25]; that is to say, the phosphorylation of MLC 2 by ROCK induces hyperactivation of actomyosin and leads to dissociation-induced apoptosis. As its name suggests, cancer cells of DGC spread from the epithelial layer and diffuse into gastric stromal tissue. Similarly to ES and iPS cells, inactivation of ROCK might protect these vulnerable cancer cells from apoptotic cell death.
The inactivation of ROCK signaling induced by RHOA mutations promoted not only cell survival but also cell migration. RHOA mutations decreased the accumulation of actin stress fiber around cell clusters and reduced intercellular adhesion, thus loosening the aggregation of cells. These morphological changes might promote cell migration. This possibility is supported by a report that diminished cell-cell interaction by actomyosin was an important step for collective cell migration, the phenomenon by which a group of cells move in concert without completely losing their cell-cell attachment [26]. Several reports that have investigated the relationship between RHOA and cell motility showed that activation of RHOA by overexpression of WT or the constitutive active form $(\mathrm{G} 14 \mathrm{~V})$ promoted cell migration [27, 28]. In contrast, our results showed that the introduction of WT and G14V decreased cell migration, and the dominant-negative mutation (T19N) enhanced cell migration (Supplementary Figure 7). To find out how the different patterns of actin stress fiber accumulation affect cell migration, further time-dependent and cell-type-dependent analyses will be necessary.

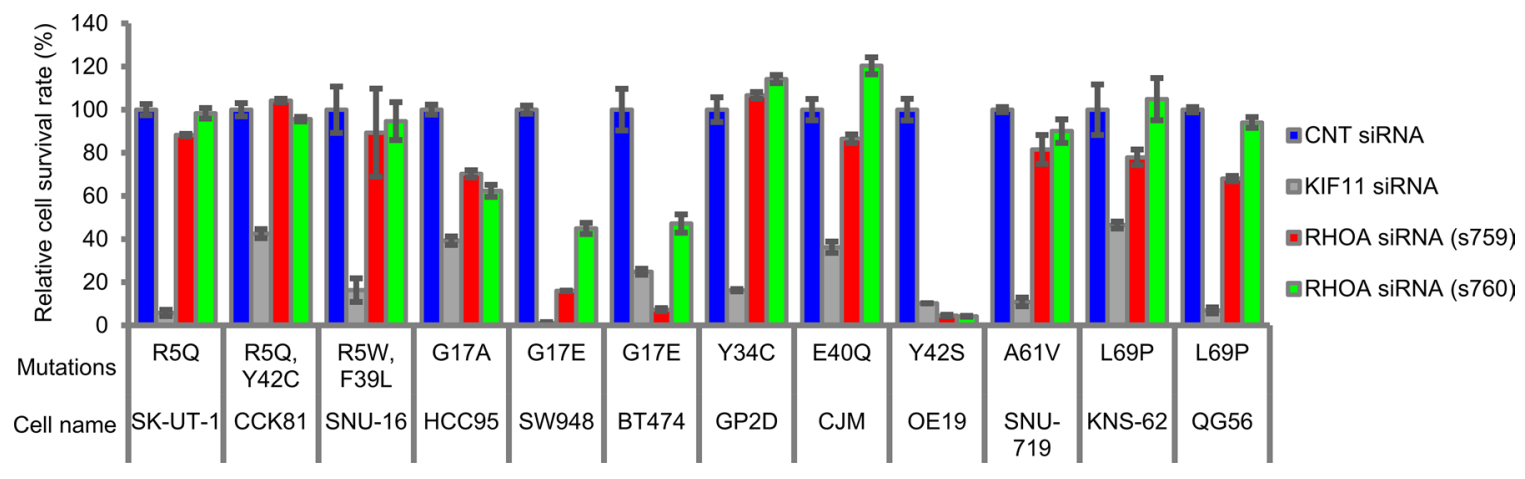

Figure 1: Cell survival rate of various types of cancer cell lines treated with $\boldsymbol{R H O A}$-siRNAs. Endogenous $R H O A$-mutated cancer cell lines were seeded in a low attachment plate and then treated with each siRNA for 7 days. The viable cells were measured by CellTiter-Glo 3D Cell Viability Assay. Data are shown as mean $\pm \mathrm{SD}(n=3)$. Cell selection criteria (see Materials \& Methods) ensured the knockdown efficiency of siRNAs.

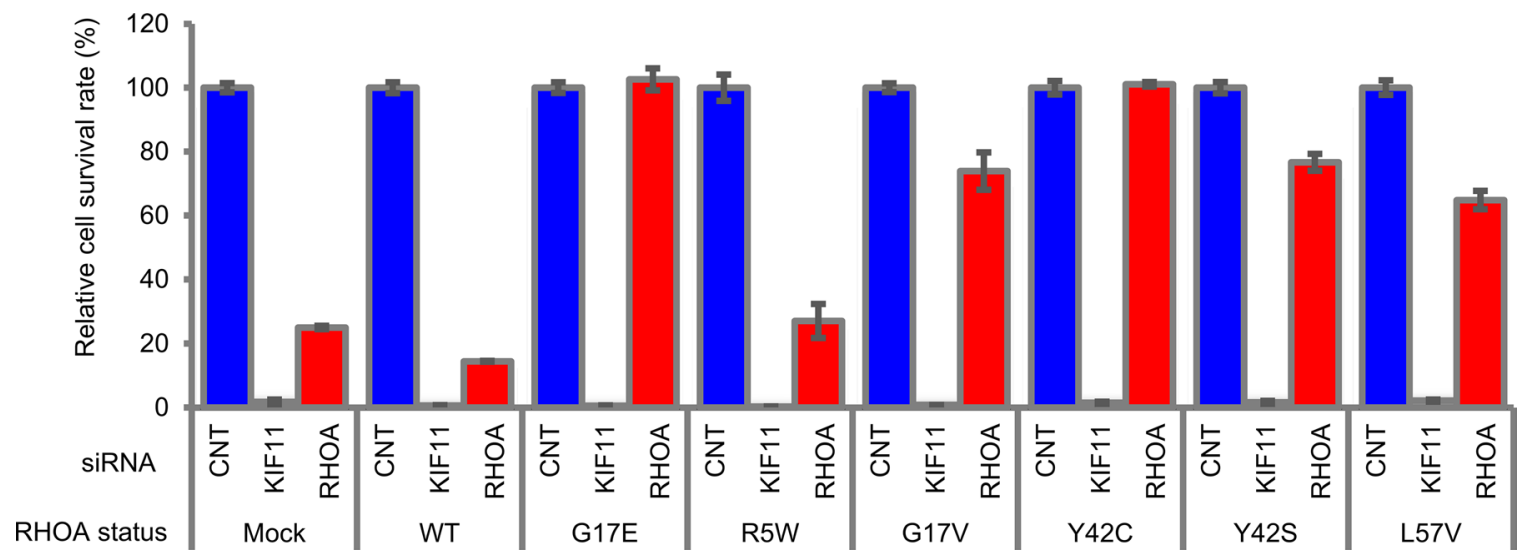

Figure 2: Rescue study of $\boldsymbol{R H O A}$-siRNA-dependent inhibition of cell survival in SW948. SW948 was transfected with WT and each mutated RHOA. Cell survival rate of obtained transfectants was evaluated as described in Figure 1. siRNA ID: s759 was used for RHOA-siRNA. Data are shown as mean $\pm \mathrm{SD}(n=3)$. Protein expression levels are shown in Supplementary Figure 8A. 
In this study, we revealed that $C L G$ fusions and RHOA mutations share a functional relationship; namely, in promoting cancer cell survival and migration. Our mutagenesis experiments showed that the GAP domain was critical for the function of $C L G$ fusions. Originally, GAPs have a BAR domain, which works as a feedback

A

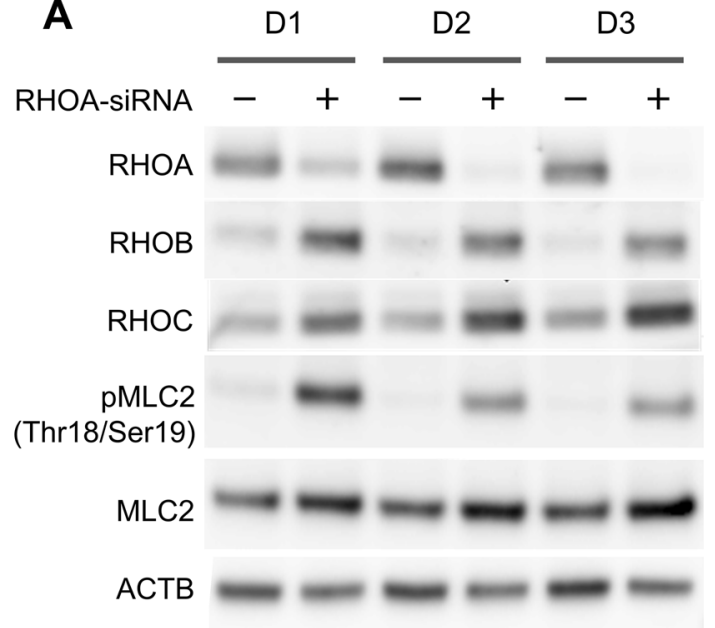

B

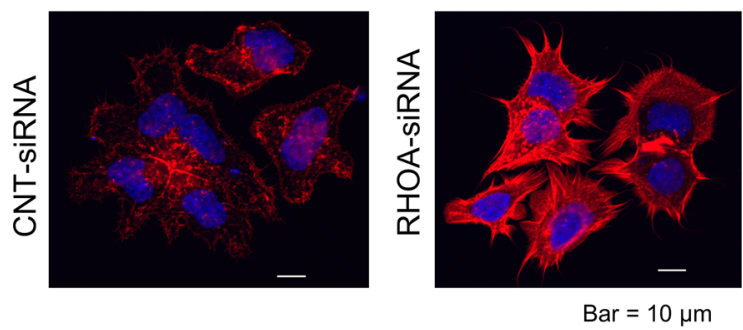

C

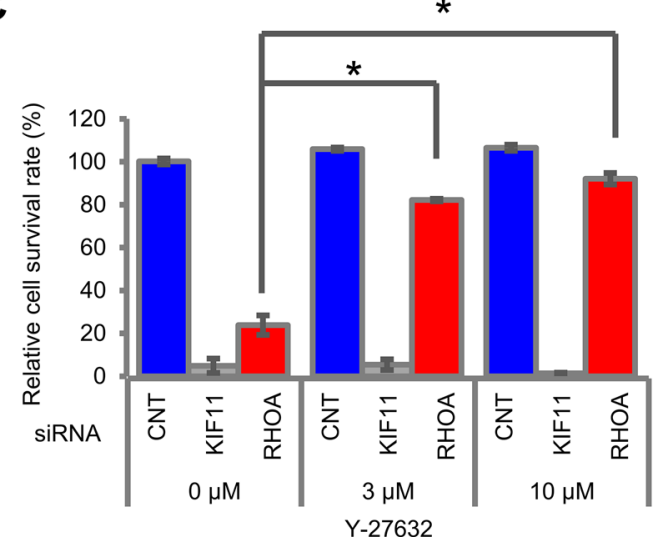

mechanism to suppress over-activated GAP activity [29], but $C L G$ fusions lose their BAR domain. So we assumed that $C L G$ fusions promote hydrolysis of GTP-RHOA to GDP-RHOA and thus inactivate ROCK signaling. Since RHOA mutations and CLG fusions are both DGCspecific genetic alterations and are mutually exclusive,

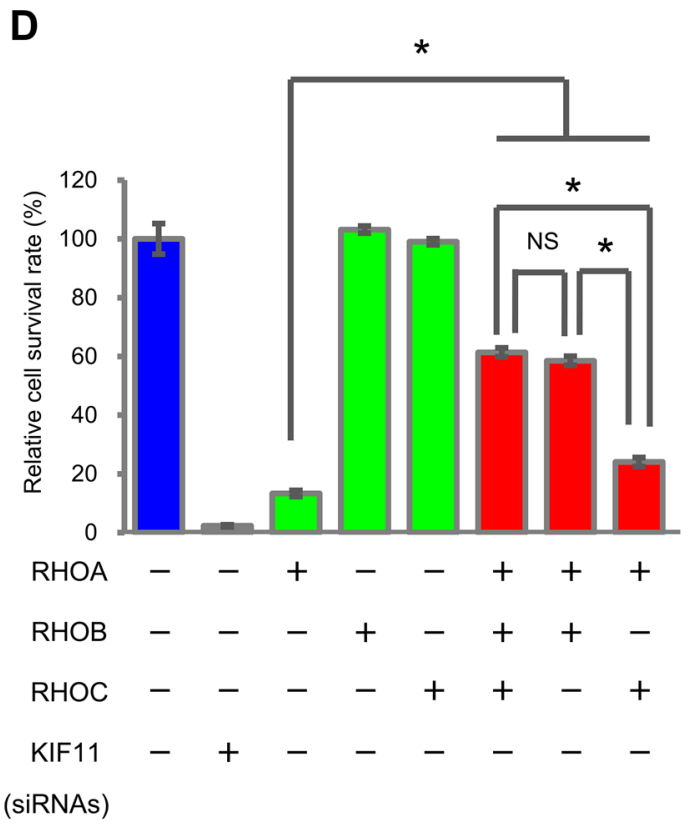

\section{E}

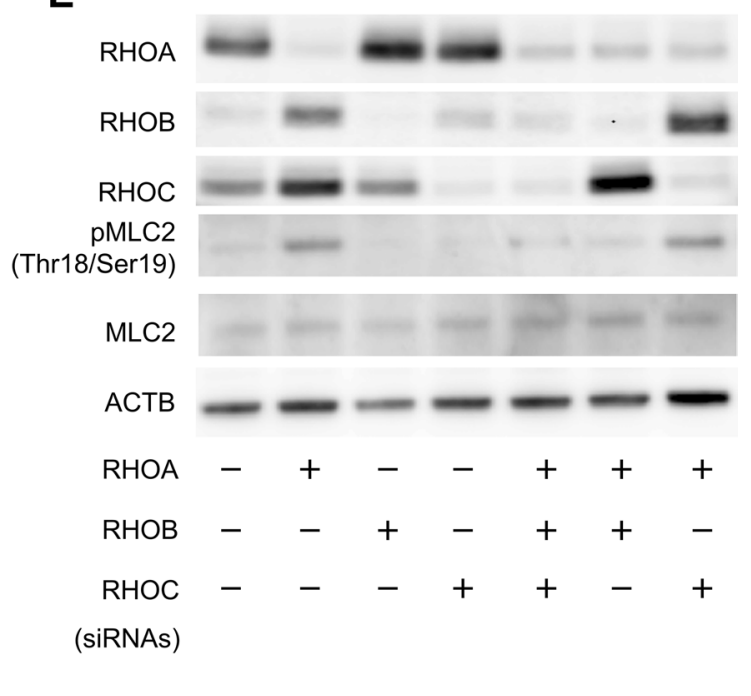

Figure 3: Activation of ROCK signaling by RHOA knockdown in SW948. (A) Expression of RHOA, RHOB, and RHOC, and phosphorylation of MLC2 in SW948 treated with $1 \mathrm{nM}$ of $R H O A$-siRNA. Proteins were harvested on days 1, 2, and 3 after siRNA treatment. The protein expression levels were detected using western blotting. (B) Actin stress fibers of SW948 treated with $1 \mathrm{nM}$ of RHOA-siRNA. Actin stress fibers were stained with Rhodamine Phalloidin, and DAPI was used for nuclear staining. Stained cells were analyzed with confocal fluorescence microscopy. Representative images of three independent chambers are shown. Details of immunocytochemistry are described in Materials and Methods. Scale bar shows $10 \mu \mathrm{m}$. (C) Restoration of cell survival by a ROCK inhibitor, Y-27632. SW948 was cultivated for 7 days with $3 \mu \mathrm{M}$ or $10 \mu \mathrm{M}$ of Y-27632. The relative cell survival rate is shown as a percentage of that in the control-siRNAtreated SW948 that was not treated with Y-27632. Data are shown as mean \pm SD $(n=3)$. Significance compared with the Y-27632 nontreated group between $R H O A$-siRNA-treated groups was determined by Student's $t$-test. ${ }^{*} p<0.05$. (D) Restoration of cell survival by $R H O B /$ RHOC-siRNAs. SW948 was cultivated for 7 days with $R H O A$-siRNA $(1 \mu \mathrm{M})$ and/or $R H O B$-siRNA $(1 \mu \mathrm{M})$ and/or $R H O C$-siRNA $(0.2$ $\mu \mathrm{M})$. Cell survival rate of obtained transfectants was evaluated as described in Figure 1. Data are shown as mean $\pm \operatorname{SD}(n=3)$. Significant differences between $R H O$-siRNA-treated groups were determined by Student's $t$-test. ${ }^{*} p<0.05$. (E) Protein expression of cells tested in (D). 
the inactivation of ROCK signaling would be a key step in the development of DGC. A ROCK signaling activator might show broad therapeutic opportunities for ROCKinactivated DGC patients.

\section{MATERIALS AND METHODS}

\section{Cell lines}

The human cancer cell lines SK-UT-1, SNU-16, SW948, and BT474 were obtained from the American Type Culture Collection (ATCC); HCC95, SNU-719, SNU-484 and SNU-638 from Korean Cell Line Bank (KCLB); GP2D and OE19 from the European Collection of Animal Cell Cultures (ECACC); CCK81, KNS-62, MKN45 and MKN74 from the Japanese Collection of Research Bioresources (JCRB); CJM from Riken; and QG-56 from IBL. Each cell line was cultured using the medium recommended by the suppliers and maintained in a humidified incubator at $37^{\circ} \mathrm{C}$ with $5 \% \mathrm{CO}_{2}$, except for SW948 cells, which were cultured without $\mathrm{CO}_{2}$.

\section{Generation of SW948 and MKN74 cell lines expressing RHOA mutants or $C L G$ fusion genes}

For the rescue studies, silencing mutations were introduced into the RHOA coding sequence (NCBI RefSeq Sequence: NM_001664.3) so that introduced RHOA were

A

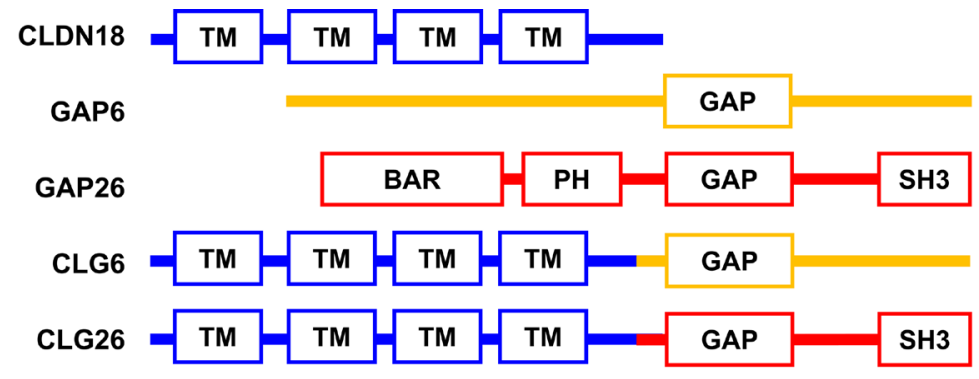

B

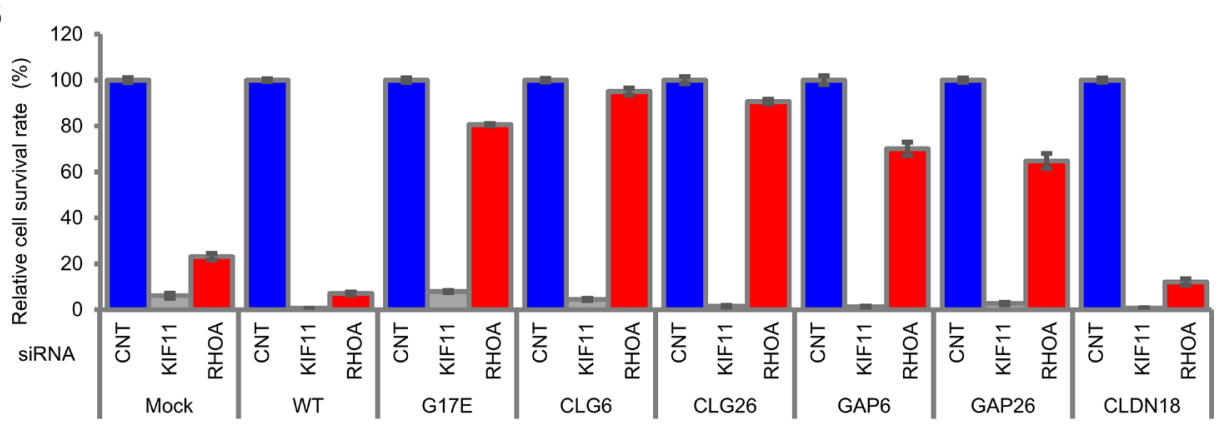

C

Human RHOA-GAP26 interaction

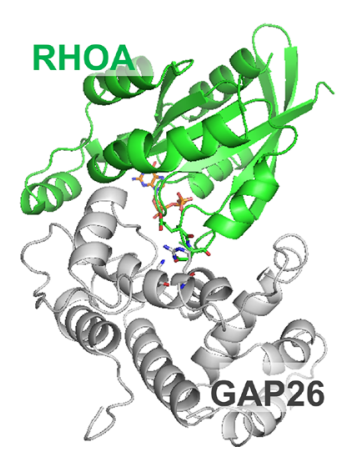

D
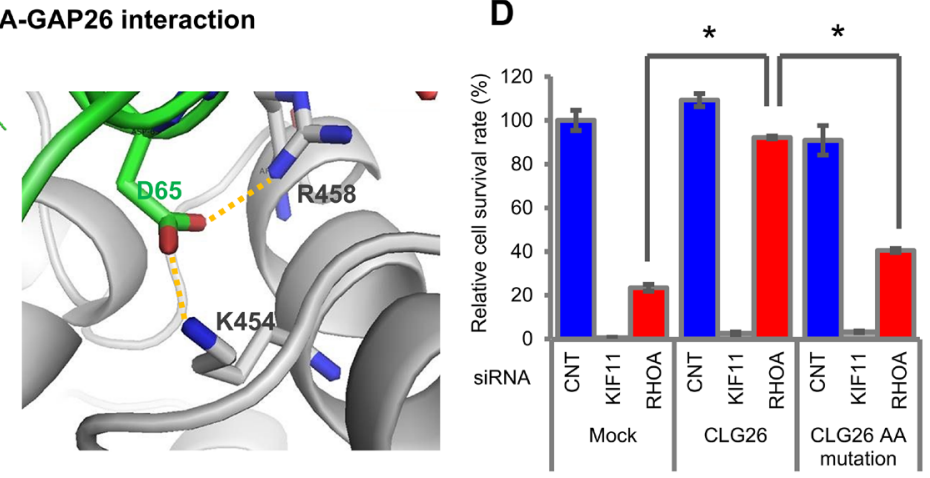

Figure 4: Cell survival promoted by $C L G$ fusions in SW948. (A) Domain structure of CLG fusions. (B) Restoration of cell survival by $C L G$ fusions. Cell survival rate was evaluated as described in Figure 3C. (C) Structure of RHOA and GAP complex inferred from homology modeling of PDB: 1TX4. RHOA in green and GAP26 in silver are shown in stick form. Hydrogen bonds are shown by an orange dotted line. A close-up (right) of hydrogen bonds in the overall model (left) is shown. (D) Reduction in cell survival activity by GAP domain AA mutations. SW948 was transfected with K454A/R458E double-mutated CLG26. Cell survival rate of each obtained transfectant was evaluated as described above. The relative cell survival rate is shown as a percentage of that in the mock transfectants treated with control-siRNA. Data are shown as mean $\pm \mathrm{SD}(n=3)$. Statistical significance of the CLG26 group compared with $R H O A$-siRNA-treated groups was determined by Student's $t$-test. " $p<0.05$. 

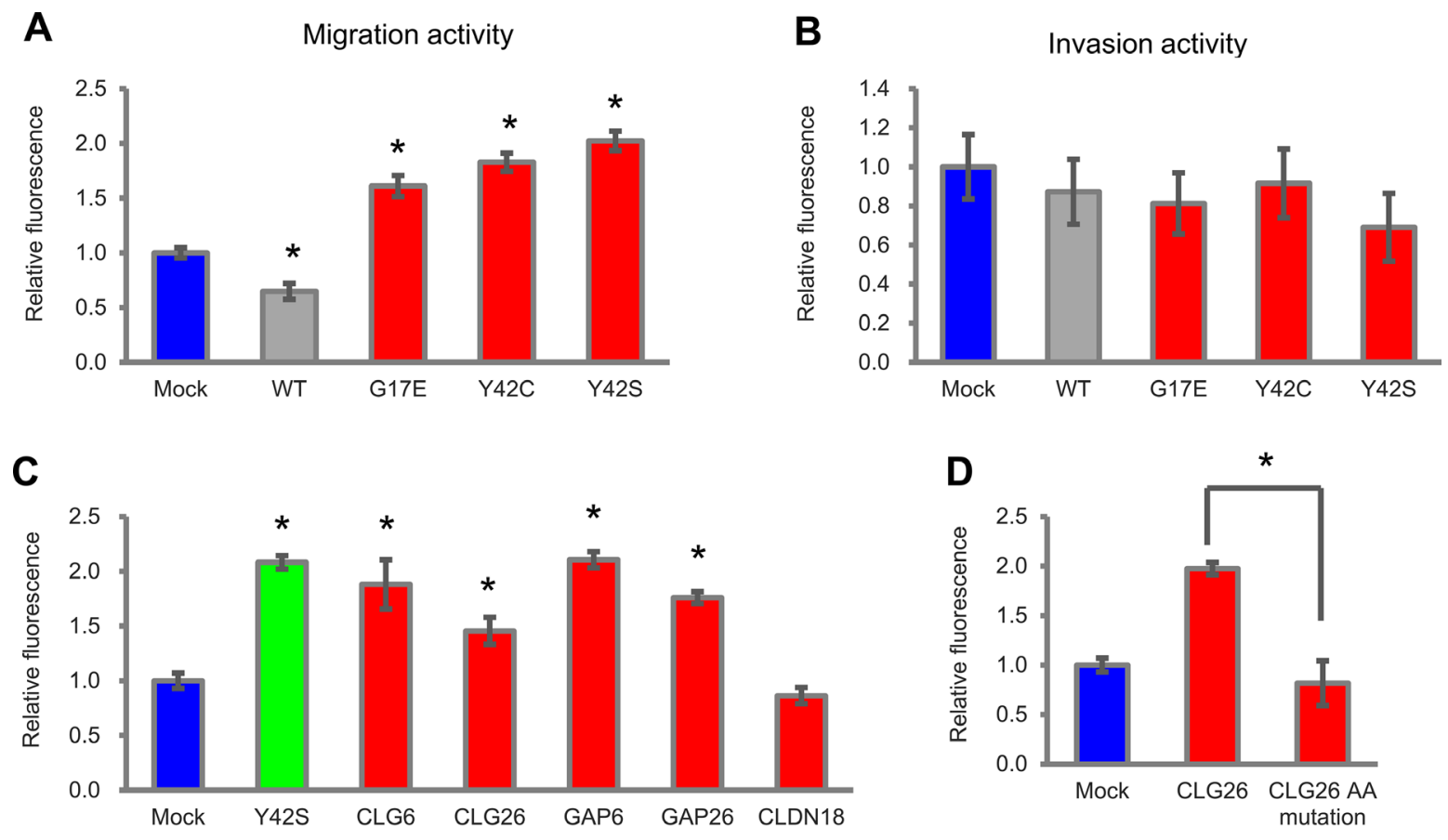

\section{E}
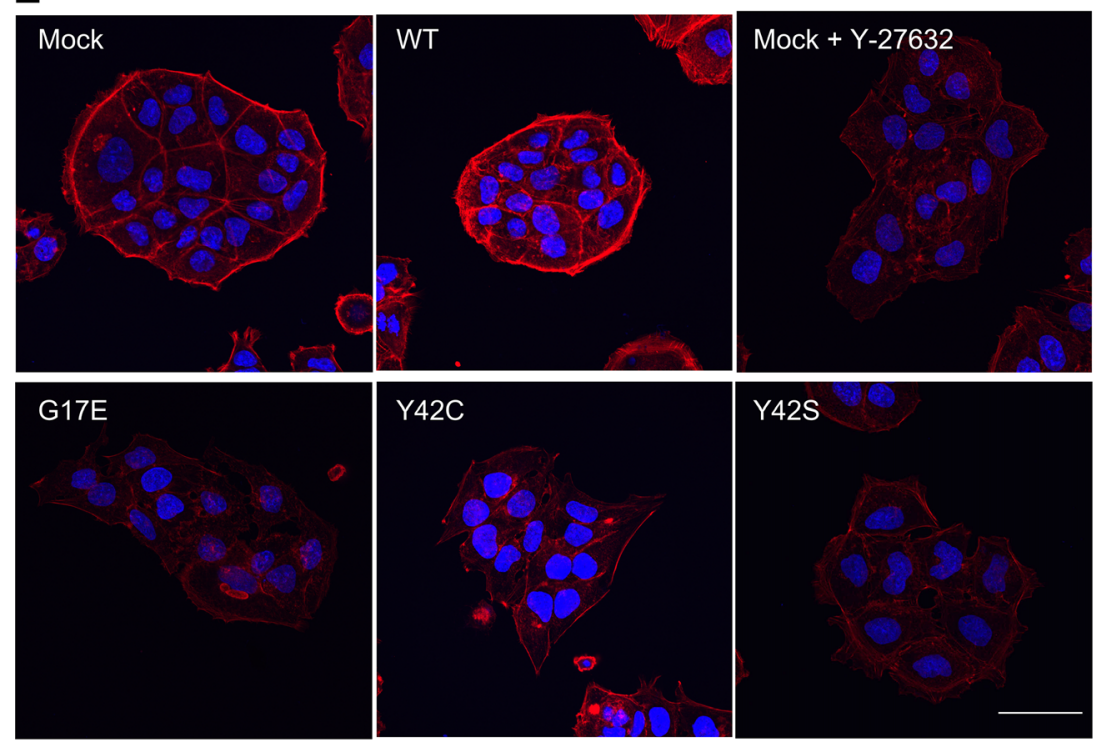

$\mathbf{F}$

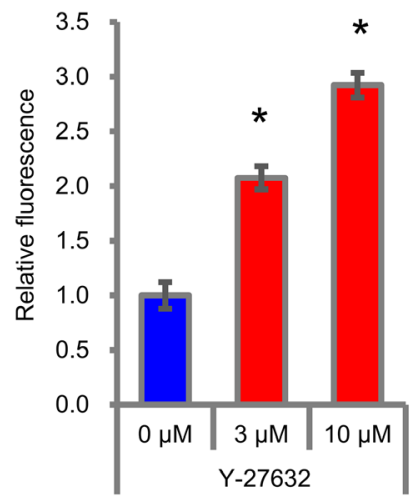

Figure 5: Cell motility in MKN74 cells with $R H O A$ mutations and $\boldsymbol{C L} \boldsymbol{G}$ fusions. (A) Cell motility in an uncoated chamber and (B) in a Matrigel-coated chamber was measured $48 \mathrm{hrs}$ after plating with MKN74 transfectants of RHOA mutations. The migrating cells were stained with calcein AM, and the fluorescence was measured by a plate reader. Data are shown as mean $\pm \operatorname{SD}(n=3)$. Statistical significance compared with the mock group was determined by Student's $t$-test. " $p<0.05$. Methods of calculating invasion activity are described in Materials and Methods. (C) Migration activity of MKN74 transfectants with CLG fusions, GAPS, and CLDN18. Cells were seeded in an uncoated chamber and migrated cells were stained with calcein AM. Data are shown as mean \pm SD $(n=3)$. Statistical significance compared with the mock group was determined by Student's $t$-test. ${ }^{*} p<0.05$. (D) Reduction of cell migration by GAP domain AA mutations. MKN74 was transfected with K454A/R458E double-mutated CLG26. Migration activity was evaluated as described above. (E) Actin stress fibers in MKN74 transfectants with RHOA mutations. Mock cells were treated with $10 \mu \mathrm{M}$ of Y-27632. Actin stress fibers were stained with Rhodamine Phalloidin, and DAPI was used for nuclear staining. Stained cells were analyzed with confocal fluorescence microscopy. Representative images from three independent fields of view are shown. Scale bar shows $50 \mu \mathrm{m}$. (F) Migration activity promoted by a ROCK inhibitor in MKN74 transfectants. Cells were seeded in an uncoated chamber and cultivated for 48 hrs with $3 \mu \mathrm{M}$ or $10 \mu \mathrm{M}$ of Y-27632. Migration activity was measured as described above. Data are shown as mean $\pm \mathrm{SD}(n=3)$. Statistical significance compared with the non-treated group was determined by Student>s $t$-test. ${ }^{*} p<0.05$. 
resistant to RHOA-siRNAs. cDNA of CLDN18 (NCBI RefSeq Sequence: NM_001002026.2), GAP6 (NCBI RefSeq Sequence: NM_013423.2), and GAP26 (NCBI RefSeq Sequence: NM_001135608.1) coding sequences was amplified in mutation-negative cancer cell lines or a cDNA library of normal human tissue (Ambion). cDNA of CLG26 fusion gene was amplified by RT-PCR from a fusion-positive gastric cancer clinical specimen. The synthesis of CLG6 fusion genes that combined cDNAs of CLDN18 and GAP6 was referred from a published report [8]. CLDN18 was fused to GAP6 and GAP26 that included the GAP domain. These sequences were inserted into the pLVSIN-CMV vector (Takara). Expression plasmids for each RHOA mutant and for CLG26 mutant with GAP domain were generated using site-directed mutagenesis PCR and the In-Fusion HD Cloning system (Clontech). The mixture of expression vector and ViraPower Lentiviral Packaging Mix (Thermo Fisher) was introduced into Lenti-X 293T cells (Takara) using FuGENE HD Transfection Reagent (Promega). After $48 \mathrm{hrs}$, the culture medium was harvested and virus particles were concentrated with Lenti-X Concentrator (Takara). Prepared lentivirus was transfected into each cell line with hexadimethrine bromide (final $8 \mathrm{ug} / \mathrm{mL}$ ). Hygromycin was added to establish stable transfectants at a final concentration of $500 \mu \mathrm{g} / \mathrm{mL}$ for SW948 and $25 \mu \mathrm{g} /$ $\mathrm{mL}$ for MKN74. After $48 \mathrm{hrs}$ of RHOA-siRNA treatment, protein expression of the siRNA-resistant RHOA was confirmed by Western blot analysis (results are shown for SW948 in Supplementary Figure 8A and for MKN74 in Supplementary Figure 8B), except for G17E/V, which was confirmed by quantitative RT-PCR (qRT-PCR), because protein expression was faint (Supplementary Figure $8 \mathrm{C}$ ). As for $C L G$ fusion genes, expression was validated by qRT-PCR (results are shown for SW948 in Supplementary Figure 9A, 9B and for MKN74 in Supplementary Figure 9C, 9D, 9E).

\section{Inhibition and rescue assays of cell survival in 3D conditions}

An assay to evaluate the inhibition of cell survival in siRNA-treated cells and a function rescue assay were performed as described previously [6]. In brief, cells were seeded in 96-well ultra-low attachment plates (Corning) in triplicate wells. At the same time, mixtures of siRNA and Lipofectamine RNAiMAX reagent (Thermo Fisher) were added to each well as 0.5 or 1 or $5 \mathrm{nM}$ of siRNA solutions. The sequences of siRNAs are listed in Supplementary Table 2A. As a non-targeting negative control siRNA, Silencer Select Negative Control No.1 siRNA (Thermo Fisher) was used. The investigation of RHOA mutated cancer cell lines was utilized public database; CCLE; https://portals.broadinstitute.org/ccle/home and COSMIC; http://cancer.sanger.ac.uk/cosmic. The 12 cell lines shown in Figure 1 were selected based on three criteria; namely, knockdown efficiency of RHOA-siRNA (over 75\%), cell survival inhibition activity by KIF11-siRNA (over 70\%), and mutation status, which was confirmed in-house. Each cell line had heterogeneous mutated and WT RHOA. In RHOA double-mutated cells (KOSC-2, CCK-81, and SNU-16), each mutation existed on different alleles. The viable cells were measured 7 days after siRNA transfection using the CellTiter-Glo 3D Cell Viability Assay (Promega). In the rescue assay, cell survival inhibition assays were performed in SW948 cell lines using siRNA-resistant RHOA or treatment with Y-27632 or RHOB/RHOCsiRNAs. We confirmed Y-27632 up to $33 \mu \mathrm{M}$ did not affect SW948 cell survival (Supplementary Figure 10A). For transient expression, we inserted each mutated $R H O A$ into a pEBMulti-Neo vector (Wako). Each plasmid was transfected into SW948 cells by electroporation with the Nucleofector system (Lonza). Then the procedure described above was followed. Protein expression in the rescue study is shown in Supplementary Figure 2A.

\section{qRT-PCR}

Cells were seeded in 6-well ultra-low attachment plates (Corning). Total RNA was extracted using the RNeasy Mini Kit (Qiagen). To evaluate RHOA, RNAs were extracted after 2 days of $R H O A$-siRNA treatment, and to evaluate $C L G$ fusion genes, RNAs were extracted after 2 days of cell seeding. qRT-PCR was performed with Power SYBR Green PCR Master Mix (Applied Biosystems), using the primers. The sequences of primers are listed in Supplementary Table $2 \mathrm{~B}$. PCR reactions were performed at $48^{\circ} \mathrm{C}$ for $30 \mathrm{~min}$ and $95^{\circ} \mathrm{C}$ for $10 \mathrm{~min}$, followed by 40 cycles of $95^{\circ} \mathrm{C}$ for $15 \mathrm{sec}$ and $60^{\circ} \mathrm{C}$ for $1 \mathrm{~min}$. Values obtained in qRT-PCR were normalized with RPS18.

\section{Western blot analysis}

Two days after transfection, cells were also lysed in RIPA buffer (Wako) supplemented with a protease inhibitor cocktail (Roche) and the phosphatase inhibitor PhosSTOP (Roche), and concentrations of the extracts were estimated with a DC protein assay (Bio-Rad). Total cell extract (3-5 $\mu \mathrm{g}$ of protein per lane) was subjected to sodium dodecyl sulfate polyacrylamide gel electrophoresis, and the separated proteins were electrophoretically transferred to Immobilon-P membranes (Millipore). After blocking in Blocking One (Nacalai Tesque), the membranes were incubated in primary antibodies against RHOA, RHOB, RHOC, phospho-CFL1 (Ser3), CFL1, phospho-MLC2 (Thr18/Ser19), MLC2, phospho-LIMK1 (Thr508)/LIMK2 (Thr505), LIMK1, LIMK2, phospho-MYPT1 (Thr696), phospho-MYPT1 (Thr853), MYPT1 (Cell Signaling, \#2117, \#2098, \#3430, \#3313, \#5175, \#3674, \#8505, \#3841, \#3842, \#3845, \#5163, \#4563, \#8574), and АCTB (Sigma-Aldrich, A1978). ACTB was used as an internal control. 


\section{Structural analysis}

The homology modelling of RHOA-GAP26 was constructed from an X-ray crystallographic structure of the RHOA-GAP1 complex (PDB: 1TX4; https://www. rcsb.org/pdb/home/home.do) using MOE2014 software (Chemical Computing Group). The figure of the RHOAGAP26 complex was drawn using PyMol v4.2.0 software (Schrödinger).

\section{Migration and invasion assay}

The MKN74 cell line was selected because it originated as differentiated gastric cancer, had RHOAWT with no clear oncogenes (e.g. KRAS, FGFR2, HER2 etc.), and was easy to handle for transfection. FluoroBlok Multiwell Insert Systems with an 8- $\mu \mathrm{m}$ pore size (Corning) were used to perform the cell migration assay for MKN74 transfectants. The cells were seeded on top of the filter inserts in $1 \%$ FBS medium. Then the inserts were placed into the lower chamber, which was loaded with $10 \%$ FBS medium. Following incubation for $48 \mathrm{hrs}$, the cells that traversed the filter were stained with calcein AM (Dojindo), and the fluorescence was read by EnVision Multilabel Reader (PerkinElmer). Stained cells were analyzed with an IX83 Inverted Microscope (Olympus) using a UPLFLN 4X $\mathrm{PH}$ objective lens. Photo data processing was performed by Olympus cellSens Dimension software ver 1.15 (Olympus). For invasion, BioCoat Tumor Invasion Multiwell Plates with 8 - $\mu \mathrm{m}$ pore size (Corning) were used. Invasion plates were re-hydrated with FBS-free media at $37^{\circ} \mathrm{C}$ for $2 \mathrm{hrs}$. After that, the procedure was the same as the migration assay. Invasion activity was calculated according to the maker's protocol (invasion activity $=$ mean number of invading cells/mean number of migrating cells). We confirmed Y-27632 up to $100 \mu \mathrm{M}$ did not affect MKN74 cell survival (Supplementary Figure 10B).

\section{Immunocytochemistry and confocal microscopy}

Cells were seeded on Nunc Lab-Tek II CC2 Chamber Slide Systems (Thermo Fisher) and either siRNAs were added simultaneously or Y-27632 was added after $24 \mathrm{hrs}$ incubation. $48 \mathrm{hrs}$ later, cells were fixed with $4 \%$ PFA, permeabilized in $0.5 \%$ Triton X-100/PBS, and stained with Rhodamine Phalloidin (Thermo Fisher). DAPI was used for nuclear staining. Stained cells were analyzed with the A1 confocal fluorescence microscopy system (Nikon) using a CFI Apochromat Lambda S 60x Oil lens. Photo data processing was performed by NISElements software (Nikon).

\section{Abbreviations}

DGC: diffuse type gastric cancers; GEF: guanine nucleotide exchange factor; GAP: GTPase-activating protein; ROCK: Rho-associated protein kinase; MLC2: Myosin regulatory light chain 2; MYPT1: myosin phosphatase targeting subunit 1; LIMK: LIM domain kinase; CFL1: Cofilin-1; CLDN: Claudin; CLG: CLDN18ARHGAP; ACTB: Beta-actin; TM: transmembrane; LoF: loss-of-function; RHO-GDI: RHO GDP-dissociation inhibitor; WT: wild type.

\section{Author contributions}

TN designed the study. TN, AH and MK performed the cell line experiments. KN performed the immunocytochemistry. TN, NK and MS drafted the manuscript. SF, SI and HA supervised and supported the study. All authors read and approved the final manuscript.

\section{ACKNOWLEDGMENTS}

We thank R. Yamoto, Y. Iguchi, and T. Takeda for the in vitro survival assays and migration assays; $\mathrm{K}$. Kashio for the actin filaments staining; Y. Watanabe for structural analysis.

\section{CONFLICTS OF INTEREST}

$\mathrm{TN}, \mathrm{KN}, \mathrm{AH}, \mathrm{SF}$, and MS are employees of Forerunner Pharma Research Co., Ltd.

\section{FUNDING}

This study was partly supported by Practical Research for Innovative Cancer Control program from Japan Agency for Medical Research and Development (AMED). (HA and SI).

\section{REFERENCES}

1. Hu B, El Hajj N, Sittler S, Lammert N, Barnes R, Meloni-Ehrig A. Gastric cancer: Classification, histology and application of molecular pathology. J Gastrointest Oncol. 2012; 3:251-61. https://doi.org/10.3978/j.issn.2078-6891.2012.021.

2. Chiaravalli AM, Klersy C, Vanoli A, Ferretti A, Capella C, Solcia E. Histotype-based prognostic classification of gastric cancer. World J Gastroenterol. 2012; 18:896-904. https://doi.org/10.3748/wjg.v18.i9.896.

3. Chen YC, Fang WL, Wang RF, Liu CA, Yang MH, Lo SS, Wu CW, Li AF, Shyr YM, Huang KH. Clinicopathological variation of lauren classification in gastric cancer. Pathol Oncol Res. 2016; 22:197-202. https://doi.org/10.1007/ s12253-015-9996-6.

4. Lazăr DT, Sporea I, Dema A, Cornianu M, Lazăr E, Goldiş A, Vernic C. Gastric cancer: correlation between clinicopathological factors and survival of patients. II. Rom J Morphol Embryol. 2009; 50:185-94. 
5. Piazuelo MB. Gastric cancer: overview. Colomb Med. 2013; 44:192-201.

6. Kakiuchi M, Nishizawa T, Ueda H, Gotoh K, Tanaka A, Hayashi A, Yamamoto S, Tatsuno K, Katoh H, Watanabe Y, Ichimura T, Ushiku T, Funahashi S, et al. Recurrent gain-offunction mutations of RHOA in diffuse-type gastric carcinoma. Nat Genet. 2014; 46:583-87. https://doi.org/10.1038/ng.2984.

7. Wang K, Yuen ST, Xu J, Lee SP, Yan HH, Shi ST, Siu HC, Deng S, Chu KM, Law S, Chan KH, Chan AS, Tsui WY, et al. Whole-genome sequencing and comprehensive molecular profiling identify new driver mutations in gastric cancer. Nat Genet. 2014; 46:573-82. https://doi. org/10.1038/ng.2983.

8. Bass AJ, Thorsson V, Shmulevich I, Reynolds SM, Miller M, Bernard B, Hinoue T, Laird PW, Curtis C, Shen H, Weisenberger DJ, Schultz N, Shen R, et al, and Cancer Genome Atlas Research Network. Comprehensive molecular characterization of gastric adenocarcinoma. Nature. 2014; 513:202-09. https://doi.org/10.1038/nature13480.

9. Wennerberg K, Rossman KL, Der CJ. The Ras superfamily at a glance. J Cell Sci. 2005; 118:843-46. https://doi. org/10.1242/jcs.01660.

10. Stankiewicz TR, Linseman DA. Rho family GTPases: key players in neuronal development, neuronal survival, and neurodegeneration. Front Cell Neurosci. 2014; 8:314. https://doi.org/10.3389/fncel.2014.00314.

11. Jaffe AB, Hall A. Rho GTPases: biochemistry and biology. Annu Rev Cell Dev Biol. 2005; 21:247-69. https://doi. org/10.1146/annurev.cellbio.21.020604.150721.

12. Tönges L, Koch JC, Bähr M, Lingor P. ROCKing Regeneration: Rho kinase inhibition as molecular target for neurorestoration. Front Mol Neurosci. 2011; 4:39. https:// doi.org/10.3389/fnmol.2011.00039.

13. O'Connor K, Chen M. Dynamic functions of RhoA in tumor cell migration and invasion. Small GTPases. 2013; 4:14147. https://doi.org/10.4161/sgtp.25131.

14. Jelen F, Lachowicz P, Apostoluk W, Mateja A, Derewenda ZS, Otlewski J. Dissecting the thermodynamics of GAPRhoA interactions. J Struct Biol. 2009; 165:10-18. https:// doi.org/10.1016/j.jsb.2008.09.007.

15. Pylayeva-Gupta Y, Grabocka E, Bar-Sagi D. RAS oncogenes: weaving a tumorigenic web. Nat Rev Cancer. 2011; 11:761-74. https://doi.org/10.1038/nrc3106.

16. Prior IA, Lewis PD, Mattos C. A comprehensive survey of Ras mutations in cancer. Cancer Res. 2012; 72:2457-67. https://doi.org/10.1158/0008-5472.CAN-11-2612.

17. Kawazu M, Ueno T, Kontani K, Ogita Y, Ando M, Fukumura K, Yamato A, Soda M, Takeuchi K, Miki Y, Yamaguchi H, Yasuda $\mathrm{T}$, Naoe T, et al. Transforming mutations of RAC guanosine triphosphatases in human cancers. Proc Natl Acad Sci USA. 2013; 110:3029-34. https://doi.org/10.1073/pnas.1216141110.

18. Watson IR, Li L, Cabeceiras PK, Mahdavi M, Gutschner T, Genovese G, Wang G, Fang Z, Tepper JM, Stemke-Hale K, Tsai KY, Davies MA, Mills GB, Chin L. The RAC1 P29S hotspot mutation in melanoma confers resistance to pharmacological inhibition of RAF. Cancer Res. 2014; 74:4845-52. https://doi. org/10.1158/0008-5472.CAN-14-1232-T.

19. Vogelstein B, Papadopoulos N, Velculescu VE, Zhou S, Diaz LA Jr, Kinzler KW. Cancer genome landscapes. Science. 2013; 339:1546-58. https://doi.org/10.1126/science.1235122.

20. O’Hayre M, Inoue A, Kufareva I, Wang Z, Mikelis CM, Drummond RA, Avino S, Finkel K, Kalim KW, DiPasquale G, Guo F, Aoki J, Zheng Y, et al. Inactivating mutations in GNA13 and RHOA in Burkitt's lymphoma and diffuse large B-cell lymphoma: a tumor suppressor function for the Galpha13/RhoA axis in B cells. Oncogene. 2016; 35:377180. https://doi.org/10.1038/onc.2015.442.

21. Ho TT, Merajver SD, Lapiere CM, Nusgens BV, Deroanne CF. RhoA-GDP regulates RhoB protein stability. Potential involvement of RhoGDIalpha. J Biol Chem. 2008; 283:21588-98. https://doi.org/10.1074/jbc.M710033200.

22. Simpson KJ, Dugan AS, Mercurio AM. Functional analysis of the contribution of RhoA and RhoC GTPases to invasive breast carcinoma. Cancer Res. 2004; 64:8694-701. https:// doi.org/10.1158/0008-5472.CAN-04-2247.

23. Wheeler AP, Ridley AJ. Why three Rho proteins? RhoA, RhoB, RhoC, and cell motility. Exp Cell Res. 2004; 301:43-49. https://doi.org/10.1016/j.yexcr.2004.08.012.

24. Claassen DA, Desler MM, Rizzino A. ROCK inhibition enhances the recovery and growth of cryopreserved human embryonic stem cells and human induced pluripotent stem cells. Mol Reprod Dev. 2009; 76:722-32. https://doi. org/10.1002/mrd.21021.

25. Ohgushi M, Matsumura M, Eiraku M, Murakami K, Aramaki T, Nishiyama A, Muguruma K, Nakano T, Suga H, Ueno M, Ishizaki T, Suemori H, Narumiya S, et al. Molecular pathway and cell state responsible for dissociation-induced apoptosis in human pluripotent stem cells. Cell Stem Cell. 2010; 7:225-39. https://doi.org/10.1016/j.stem.2010.06.018.

26. Hidalgo-Carcedo C, Hooper S, Chaudhry SI, Williamson P, Harrington K, Leitinger B, Sahai E. Collective cell migration requires suppression of actomyosin at cell-cell contacts mediated by DDR 1 and the cell polarity regulators Par3 and Par6. Nat Cell Biol. 2011; 13:49-58. https://doi.org/10.1038/ncb2133.

27. Cho SG, Li D, Stafford LJ, Luo J, Rodriguez-Villanueva M, Wang Y, Liu M. KiSS1 suppresses TNFalpha-induced breast cancer cell invasion via an inhibition of RhoAmediated NF-kappaB activation. J Cell Biochem. 2009; 107:1139-49. https://doi.org/10.1002/jcb.22216.

28. Murray D, Horgan G, Macmathuna P, Doran P. NET1-mediated RhoA activation facilitates lysophosphatidic acid-induced cell migration and invasion in gastric cancer. Br J Cancer. 2008; 99:1322-29. https://doi.org/10.1038/sj.bjc.6604688.

29. Eberth A, Lundmark R, Gremer L, Dvorsky R, Koessmeier KT, McMahon HT, Ahmadian MR. A BAR domainmediated autoinhibitory mechanism for RhoGAPs of the GRAF family. Biochem J. 2009; 417:371-79. https://doi. org/10.1042/BJ20081535. 